\title{
Retraction Note: Linguistic analysis in politically correct discourse (on the English language media content)
}

Kseniya Melnikova and Alla Guslyakova

Retracted article: SHS Web of Conferences 88, 01012 (2020)

https://doi.org/10.1051/shsconf/20208801012

The Guest Editors and the Publisher have retracted this article.

Actually, the third author of the article - Lucio Giuliodori - and his affiliation (University of Perugia, Piazza dell'Università, 1, 06123 Perugia PG, Italy) - should be removed from the list of authors of the article, because the author Lucio Giuliodori has been mistakenly included in this article.

Lucio Giuliodori has no implications in this article. The authors Kseniya Melnikova and Alla Guslyakova agree to the retraction.

Request approved on January 25, 2021.

Retracted article: Erratum SHS Web of Conferences 88, 01031 (2020) https://doi.org/10.1051/shsconf/20208801031 